\title{
$»$ Fake News Media«: Der Begriff »Fake News« als rhetorisches Mittel des Framings in der politischen Kommunikation
}

\author{
Michael Oswald
}

\section{Abstract}

Etablierte Medien in westlichen Demokratien genossen eine lange Zeit ein großes Vertrauen in der Gesellschaft. Bei den meisten Bürgerinnen und Bürgern fungierte eine Art Heuristik, in der etablierte Medien als generell vertrauenswürdig galten. Berichterstattungen wurden deshalb in der Regel nicht auf ihre grundlegende Richtigkeit hinterfragt. Dieses Vertrauen ging jedoch bei einigen Menschen verloren. Bei jenem Teil der Gesellschaft wich es einer Heuristik des Misstrauens, was besonders eklatant in den USA zutage tritt. Hier reicht mitunter ein Vorwurf von »Fake News«, um einen Bericht zu diskreditieren. Dieser Wandel ist sowohl eine Reaktion auf das jeweilige Framing in den etablierten Medien als auch eine Reframing-Strategie.

\section{Einführung}

Zahlreiche wissenschaftliche Abhandlungen über »Fake News «1-Artikel, deren Einfluss, Entstehung und Verbreitung sind in den letzten Jahren erschienen. Die Aufmerksamkeit, die dem Framing über den »Fake News«Begriff geschenkt wurde, ist jedoch gering - also für jene Strategie, Medienberichte beliebig als »Fake News« zu bekritteln. Dies scheint ein strukturelles Problem zu sein: Tandoc, Lim und Ling (2018) untersuchten wissenschaftliche Artikel, die zwischen 2003 und 2017 publiziert wurden und fanden in jenen sechs verschiedene Kategorien von »Fake News «: Satire, Parodie, Fälschung, Manipulation, Propaganda und Werbung. Bis in das

1 Selbst in solchen Meta-Analysen begegnet man dem Problem, dass der Begriff »Fake News« für die meisten Fälle zu kurz greift und er wissenschaftlich nur bedingt brauchbar ist. Hierbei sind die Begriffe der Des- oder Misinformation nicht nur präziser, vor allem aber weniger vorbelastet. In dieser spezifischen Abhandlung ist »Fake News« jedoch die präferierte Bezeichnung, da mit ihr geframt wird. 
Jahr 2017 existierte demnach keine Kategorie für das Phänomen des »Fake News«-Labels.

»Fake News«, oder wissenschaftlich eigentlich präziser Misinformation, sind in etablierten Medien nichts Neues: Bereits 1835 berichtete die New York Sun in einer sechsteiligen Artikelserie über die Entdeckung von Leben auf dem Mond (Allcott \& Gentzkow, 2017). Bisweilen wurde Misinformation sogar zu einem strukturellen Problem, wie mit der Yellow Press des späten 19. Jahrhunderts. Und als 1924 der Sinowjew-Brief über die Daily Mail - eine englische Tageszeitung - verbreitet wurde, hatte dies sogar weittragende politische Implikationen. In Deutschland zeigten die HitlerTagebücher oder der Relotius-Skandal, dass selbst große Medienhäuser vor falschen Nachrichten nicht gefeit sind. Das Neue an der »Fake News«-Debatte ist das Framing mit ebenjenem Label - also die Diskreditierung der gesamten etablierten Medien über die Zuschreibung, dass sie unwahr berichten.

Mit »Fake News«-Skandalen wie um Relotius schien sich in der letzten Zeit in einem Teil der Gesellschaft nur noch ein Misstrauen zu bestätigen, das schon länger in ihr schwelt. Daher liegt nahe, dass das »Fake News«Label lediglich Teil eines größeren und grundlegenden Wandels in modernen Demokratien ist (Egelhofer \& Lecheler, 2019). Das Label ist in der Tat ein Ausdruck für das wachsende Misstrauen gegenüber den etablierten politischen Eliten, den Vertrauensverlust in das politische System, die Unzufriedenheit mit der Demokratie, die Ablehnung des progressiven Wandels in der Politischen Kultur sowie die negativen Effekte der Globalisierung. Sein Siegeszug ist jedoch nicht nur auf die politischen, sondern auch auf die technologischen Veränderungen des 21. Jahrhunderts zurückzuführen, insbesondere die Möglichkeit der direkten Kommunikation, die eine Umgehung der traditionellen Gatekeeper sowie eine ganze Palette an alternativen Medien erst möglich machte. ${ }^{2}$

Gerichtet ist das Label zunächst gegen eine Generation an Journalisten, die in der Wahrnehmung ihrer Opponenten insbesondere den Wandel in der Politischen Kultur befürworten oder gar verstärken. Ein Teil der

2 Die neue Medienstruktur macht ein Umgehen der traditionellen Gatekeeper, wie die klassischen Medien, überhaupt erst möglich (Entman \& Usher, 2018). In der Vergangenheit setzten die New York Times und die Washington Post die Agenda für Zeitungen, Fernseh- und Radioberichterstattung. Mit dem Erfolg der sozialen Medien eröffneten sich nicht nur mehr Möglichkeiten für einzelne Personen, Botschaften und Frames zu verbreiten, auch veränderten sie die Medienagenda (Entman \& Usher, 2018; Meraz, 2011; Vargo \& Guo, 2017). So nahmen Social-MediaPlattformen dem traditionellen Journalismus das Monopol der Produktion und Verbreitung von Nachrichten (Hermida, 2011; Tandoc \& Vos, 2016). 
Gesellschaft entsagte sein Vertrauen in die großen Medien, was jene Heuristik tangierte, mit welcher die Integrität der etablierten Medien in der Vergangenheit kaum hinterfragt wurde. Diese Veränderung ist primär auf zwei Gründe zurückzuführen: (1) die strategische Unterminierung der großen Presse-Outlets, (2) der zwar meist relativ schwache, aber dennoch vorhandene progressive Bias bei einigen Publikationen. Das hieraus entstandene Misstrauen macht es nun möglich, sämtliche etablierten Medien-Outlets mit dem »Fake News«-Vorwurf grundlegend zu diskreditieren.

\section{Ein neuer Frame über die Medien}

Die etablierten Medien genossen in westlichen Gesellschaften eine lange Zeit ein hohes Ansehen - die Bürgerinnen und Bürger schenkten ihnen ein großes Vertrauen. Jenes war zwar nie blind, jedoch galten die großen Nachrichten-Medien als zuverlässig, kompetent und integer (Tandoc et al., 2017). Man kann dieses Vertrauen auch als eine Art Heuristik verstehen, in der zumindest keine kognitive Energie für die Bewertung des Wahrheitsgehaltes einzelner Nachrichten aufgewendet werden musste - die Heuristik war als Grundlage der Legitimität ausreichend (Tandoc et al., 2017). Dies ist auch darin begründet, dass im heuristischen Model der Informationsverarbeitung Einstellungen vereinfacht formiert werden (Chen \& Chaiken, 1999).

Heuristiken fungieren als eine Art Denkschablone und sie dienen Menschen als präformierte Interpretationsrahmen. ${ }^{3}$ Eine angebotene Information wird dabei auf Basis von bereits etablierten Heuristiken interpretiert und mit bekannten Konzepten, Kategorien, Stereotypen etc. abgeglichen. Somit leiten und filtern jene kognitiven Verknüpfungen die Informationsverarbeitung (Hatfield-Edwards \& Shen, 2005). Auf diesem Wege wird die Realität entschlüsselt (Bateson, 1972 [1955]; Goffman, 1974). Da Heuristiken individuell vorgeprägt sind, entsteht als Folge dieses Interpretationsmechanismus eine spezifische Perspektive, in der die Wahrnehmung vereinfacht wird. Dies bringt eine gewisse Verzerrung sowie eine unterkomplexe Beurteilungsfähigkeit mit sich, da die vorstrukturierten Rahmen lediglich

3 Die Tradition, Denk-Heuristiken in Sozialwissenschaften zu beschreiben, geht auf William James zurück, der die Wahrnehmung der Umgebung für ein Baby als eine »big, blooming, buzzing confusion« (James, 1998 [1890], 16) beschreibt, weil es noch keine Denkrahmen geformt hat. Mit Gregory Bateson und Erving Goffman wurde hieraus das Konzept der Denk-Frames in die Sozialwissenschaften eingeführt und als das Konzept der Frames popularisiert (Bateson, 1972 [1955]; Goffman, 1974). 
ein Abbild der sozialen Realität liefern (Landau, Keefer \& Rothschild, 2014; Williams, 2004). Extreme dieser Vereinfachung wären einerseits das blinde Vertrauen in Aussagen, die in etablierten Medien geäußert werden; andererseits wäre das die Vermutung, dass Journalisten jenes Standes eine politische Agenda verfolgen und daher alle ihre Aussagen verzerrt sein müssen. Dieser generelle Blickwinkel auf einen Gegenstand kann als Heuristik-Frame bezeichnet werden, da ihn ein vordefinierter Rahmen umspannt.

Der Frame über die etablierten Medien war bei Bürgerinnen und Bürgern westlicher Gesellschaften größtenteils so vordefiniert, dass sie ihnen Zuverlässigkeit, Kompetenz und eine gewisse Integrität zuschrieben (Tandoc et al., 2017). Der Glaube an diese Qualität ist nicht mehr bei allen Segmenten westlicher Gesellschaften vorhanden. Bei jenen Menschen wurde der Wahrnehmungs-Frame des Vertrauens überschrieben - in eine Heuristik des Nicht-Vertrauens. So wurde das Reframing der Mainstream-Presse über das »Fake News«-Label erst möglich, da die positive, unkritische Heuristik bei diesem Teil der Gesellschaft nicht mehr existiert.

Wenn man von Diskreditierung über »Fake News« spricht, dann kommt man an dem Mann nicht vorbei, der die etablierten Medien als Feind des Volkes bezeichnet: Donald J. Trump. Zuhauf schreibt er ihnen eine politische Agenda zu und erwähnte in einem Gespräch mit Lou Dobbs von Fox Business im Oktober 2017: »I really started this whole ,Fake News" thing « (Thomsen, 2017). Trump stellte hiermit auf das Framing mit dem $\gg$ Fake News«-Label ab und obwohl er weder Urheber der Idee ist noch die Voraussetzungen dafür schuf, dass diese Strategie überhaupt funktioniert, hat er tatsächlich das Verständnis über die Mainstream-Presse erfolgreich reframed.

In den Vereinigten Staaten hegten vorwiegend konservativ geprägte Menschen gegenüber den etablierten Medien schon seit geraumer Zeit eine gewisse Skepsis. Sie sehen im Framing der Mainstream-Presse ein liberales Bias. Trump nutzte diese Skepsis und erweiterte sie hin zur Wahrnehmung, dass Mainstream-Medien generell verzerrt oder gar unwahr berichten. Zudem popularisierte er diese Technik - zumindest in westlichen Staaten. Trump baute damit lediglich auf ein Misstrauen, das sich über Jahrzehnte hinweg unter Konservativen aufgebaut hat und strategisch verstärkt wurde. 


\section{Das Label Liberal Media}

Konservative Wortführer begannen schon vor Jahrzehnten, die traditionellen Medien mit dem Label Liberal Media zu diskreditieren (Prior, 2013). Seit den 1950er Jahren war es ein Ziel, auf diesem Wege die MedienAgenda neu zu definieren. Bis in die 1960er Jahre hinein schien für das Gros der Gesellschaft kaum eine Notwendigkeit geboten, auf den Mainstream einwirken zu müssen: Bis auf den New Deal hielt sich im 20. Jahrhundert ein relativ zentristischer Konsens, der bis in die Mitte der 60er Jahre Gesellschaft und Politik dominierte. Selbst die ideologischen Unterschiede der beiden großen Parteien - die Demokraten und die Republikaner - waren marginal. Zudem wurden viele der heute relevanten sozialliberalen Issues nicht öffentlich diskutiert, die wenigsten davon wurden überhaupt diskutiert. Die relativ wenigen Medienhäuser - regionale und nationale Tageszeitungen sowie drei Fernsehsender - hatten an diesem zentristischen Konsens teil. Selbst die lizensierten Radio-Sender lieferten in jener Zeit eine Berichterstattung in einer relativ homogenen Manier, die als generisch abgewogen gelten kann (vgl. Oswald, 2018, Hahn \& Iyengar, 2009). Das kam nicht von ungefähr, denn eine ausgewogene Berichterstattung war sogar gesetzlich verankert: Mit der Fairness-Doktrin wurde es bis dahin unterbunden, einseitig zu berichten. Im Jahre 1949 war sie eingeführt worden, um sicherzustellen, dass bei kontroversen Themen verschiedene Perspektiven und Meinungen beleuchtet werden.

Gerade diese zentristisch-uniforme Ausrichtung war Autoren und Verlegern wie Henry Regnery, William Rusher, John Chamberlain, Henry Hazlitt oder William Buckley Jr. ein Dorn im Auge. Als im Winter 1955 die erste Ausgabe der National Review erschien, schrieb ihr Herausgeber Buckley im Mission Statement:

There never was an age of conformity quite like this one, or a camaraderie quite like the Liberals'. Drop a little itching powder in Jimmy Wechsler's bath and before he has scratched himself for the third time, Arthur Schlesinger will have denounced you in a dozen books and speeches, Archibald MacLeish will have written ten heroic cantos about our age of terror, Harper's will have published them, and everyone in sight will have been nominated for a Freedom Award. Conservatives in this country - at least those who have not made their peace with the New Deal, and there is serious question whether there are others - are non-licensed nonconformists; and this is dangerous business in a Liberal world, as every editor of this magazine can readily show by pointing to his scars. (Buckley, 1955, 5)

Die genannten Autoren werden nicht umsonst attackiert: Wechsler war als Chefredakteur und Kolumnist der New York Post ein Verfechter des amerikanischen Liberalismus von ähnlichem Format, wie es auch Schlesinger 
und MacLeish zugeschrieben wird. Jene Publikationen wie die National Review dienten bis in die 1960er Jahre hinein jedoch zuvorderst als Korrektiv innerhalb konservativer Kreise. Die Etablierung einer konservativen Gegensphäre wurde erst mit einem Wandel in der Politischen Kultur möglich.

In der zweiten Hälfte der 1960er Jahre setzte ein gesellschaftlicher Wandel ein, durch den sich die Politische Kultur drastisch veränderte, insbesondere durch die Bürgerrechtsbewegung und die gesellschaftlichen Umwälzungen der 1960er Jahre. Konservative alarmierten die sozialen Unruhen und sie fühlten sich aufgrund der Aufhebung des Status quo marginalisiert (Major, 2012). Dabei rückte eine zunehmend progressiv wahrgenommene Ausrichtung der nationalen Medien in das Zentrum der Kritik: Das Label Liberal Media war unter Konservativen bereits mit der Präsidentschaftskampagne um Barry Goldwater 1964 ein allgemein bekannter Begriff geworden. Nicht nur lamentierten Goldwater und seine Strategen gegen die ablehnende Berichterstattung gegenüber dem Senator von Arizona. Auch wurden Vorwürfe laut, er habe die Präsidentschaftswahl aufgrund der liberalen Tendenzen in der Presseberichterstattung verloren (Link, 2013). Unter Konservativen schien es klar zu sein, dass sich das Gros der Journalisten gegen Goldwater positionierte (Major, 2012).

Allerdings wurde in jener Zeit generell eine zunehmend progressiv perzipierte Haltung in den Medien beklagt. Diese zu kontern war eine Aufgabe, der sich Herausgeber von Zeitschriften wie des Freeman, der National Review oder von Human Events verschrieben. Deren Berichterstattung solle nicht verzerrt, jedoch durch eine konservative Perspektive geprägt sein, kündigten die Herausgeber von Human Events an:

In reporting the news, Human Events is objective; it aims for accurate representation of the facts. But it is not impartial. It looks at events through the eyes that are biased in favor of limited constitutional government, local self-government, private enterprise and individual freedom. These principles represented the bias of the Founding Fathers. We think the same bias will preserve freedom in America. (Human Events, 1964, 14)

Es dauerte auch nicht lange, bis die Presse eine wirklich breite Ablehnung aus vornehmlich konservativen Kreisen erfuhr. In den späten 1960er und insbesondere den frühen 1970er Jahren wurden ihre Vertreter bezichtigt, den Krieg in Vietnam zu einseitig und negativ darzustellen. Tatsächlich gab es in Teilen der Presse ein neues sozialliberales Moment, vor allem war dies jedoch an den Universitäten verbreitet: In einer Umfrage der Jahre 1969/70 verorteten sich Studierende zu drei Fünfteln als politisch links, nahezu 50 Prozent sprachen sich für eine Sozialisierung aller grundlegenden Industrien aus, rund 70 Prozent hätten eine unilaterale atomare Abrüstung befürwortet und 54 Prozent hätten eine Kapitulation vor der Sowjetunion im 
Falle eines Konflikts ersucht (Nash, 2006). Als die Ergebnisse dieser Erhebung 1971 in der National Review publiziert wurden, sah Lewis F. Powell einen dringenden Handlungsbedarf - an den Universitäten wurden schließlich die Eliten von morgen ausgebildet.

Lewis F. Powell Jr. verfasste im Jahre 1971 ein Memorandum, mit dem der sozialliberale Diskurs in den Medien Einhalt geboten werden sollte insbesondere der von ihm empfundenen Anfeindung von Geschäftsleuten. Er entwarf mit dem Memorandum eine Blaupause, die als Reframing-Strategie gedeutet werden kann (Oswald, 2018). Powell sah hierfür eine Restrukturierung im Mediensystem, eine Etablierung konservativer Think Tanks und einen Einfluss auf akademisch-intellektuelle Institutionen vor. Das Memorandum hatte zur Folge, dass ein Treffen mit mehr als 200 Teilnehmern einberufen wurde, darunter die 42 Vorstände von den 50 größten Industrieunternehmen in den USA (Korten, 2001). Zusammen mit den Bemühungen von William Simon, der vor allem konservative Unternehmensführer dazu brachte, seine Idee der Counterintelligentsia zu unterstützen, wurde eine finanzielle Basis geschaffen, um die perzipierte Befangenheit in den Medien zu bekämpfen. Jenes Geld beispielsweise, das von Unternehmen für Werbungen aufgeboten wurde, sollte nach Powells und Simons Vorstellungen zu Medien fließen, die ihre Interessen eher berücksichtigten. Die rechtliche Grundlage für diese neue Art von Medien schuf jedoch die Federal Communications Commission (FCC) 4 selbst, indem sie 1987 ankündigte, die Fairness-Doktrin nicht mehr durchzusetzen (Aufderheide, 1990).

Die de facto Abschaffung der Fairness-Doktrin war der Beginn einer neuen Form der Berichterstattung. Unter christlichen Amerikanern war das Talk-Radio schon seit den 1930er Jahren beliebt und Konservative sahen nunmehr die Möglichkeit, ähnlich hohe Reichweiten, wie die christlichen Sendungen erreichen zu können. Es dauerte nicht lange, bis nach 1987 das konservative Talk-Radio ein voller Erfolg wurde. Eines der prominenteren Beispiele dieser Zeit ist Rush Limbaugh, der in den späten 1980er Jahren $\mathrm{zu}$ einem der meistgehörten Radio-Moderatoren avancierte. Bis heute erreicht er zweistellige Millionen-Einschaltquoten. Nach diesem Vorbild wurden politische Sendungen mit einem spezifischen Einschlag konservativer Interpretationen etabliert. Strategen witterten dies als Erfolgs- und Investoren als Geschäftsmodell.

Auf beiden Seiten des politischen Spektrums wurden von nun an massive Investitionen im Mediensektor getätigt, insbesondere nachdem mit dem guliert. 
Telecommunications Act 1996 die Sparte enorm dereguliert wurde. Neben dem prominenten Beispiel FOX News reicht die konservative Medienlandschaft heute von $C N B C$, Weekly Standard, reason.com, Federalist, Drudge Report, Daily Wire, Breitbart bis hin zu den extrem verzerrten Infowars ${ }^{5}$ von Alex Jones. Mit einer solchen Berichterstattung wird eine konservative Interpretation von Sachverhalten oder auch die - mitunter auch fadenscheinige - Zuschreibung von Verantwortung mit dem jeweiligen Bericht mitgeliefert, so dass aktuelle Ereignisse stets in einem spezifischen Verständnis vordefiniert sind. Konservative Akteure und Institutionen wie Fox News oder Radio-Talk-Show-Hosts wie Sean Hannity oder Mark Levin befinden, dass sie als ein benötigtes Korrektiv zu den etablierten Medien dienen (Major, 2012). Mit diesem politischen Format verschwand aus vielen jener Sendungen auch das Konzept der Objektivität, unter welchem überparteilich und möglichst neutral über die Fakten berichtet wurde. Der Objektivitätsanspruch war lange ein Eckpfeiler des professionellen Journalismus in den USA gewesen (Marchi, 2012).

Nicht selten lösen sich jedoch Moderatoren und Journalisten konservativer Medien von diesem Grundsatz, auch gängeln sie die Mainstream-Medien bei nahezu jeder Gelegenheit. Hannity bezeichnet diese beispielsweise gerne als Media-Mob oder Lou Dobbs als National-Left-Wing-Media. Die Bezeichnung Mainstream-Medien als Signum des lange beschworenen sozialliberalen Bias ist mittlerweile ein wichtiger Teil der Rhetorik im Konservatismus. Damit sichern sich die Kommunikatoren auch die Deutungshoheit über Sachverhalte (Major, 2012).

Alles Sozialliberale erklärte Rush Limbaugh bereits vor zehn Jahren zur Sphäre der Falschinformation:

We live in two universes. One universe is a lie. One universe is an entire lie. Everything run, dominated, and controlled by the left here and around the world is a lie. The other universe is where we are, and that's where reality reigns supreme and we deal with it. (Limbaugh, 2009)

Eine solche Aussage wird von vielen Konservativen kaum hinterfragt. Das liegt jedoch nicht allein an der stetigen Verurteilung der Mainstream-Medien, die gewiss nicht zuletzt durch die wachsende Kritik an Vertrauen

Jones verbreitet gar krude Verschwörungstheorien, wie dass beispielsweise das Massaker an der Sandy Hook Elementary School inszeniert war und die Kinder in Video-Berichten Krisenschauspieler gewesen seien. Als Beweis diente ein Regionalpresse-Video aus Kalifornien, in dem der Sprecher einer Aktivisten-Gruppe aus der Schülerschaft des Attentats, David Hogg, als Zeuge eingeblendet wurde. Hogg war nach eigener Aussage auf einem Familienbesuch in Los Angeles. Für viele Menschen war dies jedoch der Nachweis, dass Krisenschauspieler von der Regierung engagiert wurden, um härtere Waffengesetze durchzusetzen. 
eingebüßt haben (Prior, 2013). Vielmehr sind die ideologischen Präferenzen von Konservativen und den meisten Mainstream-Journalisten so unterschiedlich, dass die Inhalte bisweilen als verzerrt oder gar unkorrekt wahrgenommen werden.

\section{Ein progressives Bias?}

Tatsächlich stehen Berichte von überregionalen Presse-Organisationen bisweilen in einem Licht, das mit der Perspektive einiger Bürgerinnen und Bürger nicht übereinstimmt (Richardson, 2017). Das Framing von Journalisten aus Washington D.C., New York, Boston oder Los Angeles spiegelt oft nicht die Lebens- und Denkweisen wider, die Menschen aus Tulsa oder Mena verkörpern. Die kulturelle Spaltung zwischen den großen Städten und ländlichen Gebieten ist groß und Journalisten, die meist im Umfeld der Ostküsten-Staaten leben, bewerten Sachverhalte oft anders als jemand aus den häufig als Fly-Over-States gescholtenen Gegenden. So ist den Medien von der New York Times bis hin zur Washington Post tatsächlich ein progressives Bias inhärent. Freilich ist dieses nicht konstant, bisweilen ist es sogar lediglich durch eine konservative Perspektive wahrgenommen.

Doch selbst ein geringes Bias sorgt in Kombination mit der Diskreditierung der Mainstream-Presse für Probleme: Konservative schätzen den Grad der Verzerrung in Medien überproportional hoch ein, wenn die Berichterstattung nicht im Einklang mit ihrer Weltanschauung steht (Feldman, 2010). Außerdem kommt es bei Menschen häufig zu einem Bestätigungsfehler (Confirmation Bias). Sie bevorzugen es, Informationen zu konsumieren, welche die eigene Sicht auf Sachverhalte bestätigen und lehnen vermehrt Nachrichten ab, welche die eigene Meinung durchkreuzen. Somit hat der Bestätigungsfehler auch einen Einfluss auf die Glaubwürdigkeit: Inhalte werden mit größerer Wahrscheinlichkeit als glaubwürdig eingestuft, wenn sie mit dem übereinstimmen, was Empfänger bereits denken (Metzger, Flanagin \& Medders, 2010). Entsprechend schätzen Amerikaner im Gesamten, dass 62 Prozent der Nachrichten in Print-, Hör- und TV-Nachrichten verzerrt sind. Unter den Anhängern der Republikaner wird die Mehrzahl der Mainstream-Medien als »Fake News« angesehen, nur Fox News ${ }^{6}$ und das Wall Street Journal betrachten sie als unverzerrt (Jones, 2018). Der

6 Wenn Konservative vor allem Fox News folgen und dem Sender das meiste Vertrauen aussprechen, bedeutet dies nicht, dass sie keine Mainstream-Medien konsumieren. Sie tun dies, weshalb die These der Filterblasen mittlerweile angezweifelt wird. 
heute unter Konservativen oftmals gehegte Verdacht, dass die MainstreamMedien im Allgemeinen liberal voreingenommen sind, könnte sie auch dazu bringen, dies ihrer Voreinstellung entsprechend wahrzunehmen. Dies würde die Vermutung einer Heuristik des Misstrauens bestätigen. In jedem Fall zeigt sich mit dieser Selbsteinschätzung, dass das jeweilige ideologische Spektrum verschiedene Erwartungen an die Medien richtet: Es soll das übersetzt werden, was zu den themenbezogenen Befindlichkeiten passt (Feldman, 2010). ${ }^{7}$

Mit dem neuen Angebot an gefilterter Darstellung änderten sich auch die Medien-Präferenzen vieler Amerikaner. Das Sendeformat mit konservativen Framings erreichte vor allem auf Fox News einen einmaligen Erfolg. So dominierte The O'Reilly Factor für über ein Jahrzehnt den Markt der Kabel-Nachrichten. Hannitys Sendung folgte auf Platz zwei (Peck, 2014). Demgegenüber verloren die öffentlichen Kanäle bis zu 50 Prozent ihrer Zuschauer innerhalb der letzten Jahrzehnte (Nielsen Media Research, 2013). In diese neue Befindlichkeit stieß Donald Trump mit seiner Anti-Establishment-Kampagne.

\section{Trump und »Fake News»}

Trump nutzte die Voreingenommenheit vieler Bürgerinnen und Bürger gegenüber den Mainstream-Medien wie kaum jemand zuvor. Die konservative Klientel nahmen sie schon länger als das wahr, was die Strategen der 1960/70er Jahre als Abbild erreichen wollten: einen verlängerten Arm der liberalen Elite (Guardino \& Snyder, 2012). Damit passte diese Ausrichtung perfekt zu Trumps Wahlkampfstrategie. Trump hat dabei das bestehende Liberal-Media-Label gekapert und es mit dem »Fake News«-Label neu besetzt. Er reframte damit nicht nur das Konzept der »Fake News«, seine neu gewonnene Deutungshoheit bietet ihm zudem den Vorteil, die Begriffe Wahrheit und Realität in das Gegenteil zu verkehren.

7 Jedoch sind es nicht nur die Konservativen, die eine eigene Sphäre geschaffen haben. Demokraten orientieren sich weiterhin an Nachrichtensendern wie $C N N$ und $N P R$, allerdings hat sich auch auf der sozialliberalen Seite eine Sphäre mit einem dezidiert sozialliberalen Einschlag etabliert, wie MSNBC, Mother Jones, The Huffington Post, bis hin zum Palmer Report. Mit dem Angebot an alternativen Medien und der Diskreditierung des Mainstreams teilte sich die Medienstruktur in den Vereinigten Staaten im Grunde parallel zur Spaltung der Gesellschaft, wobei die Henne-Ei-Frage bei dieser Entwicklung nicht eindeutig geklärt ist, also ob sich zunächst die Einstellungen verschärften und dies zu ideologisierten Medien führte oder ob die Medienstruktur die Polarisierung der Gesellschaft erst ermöglicht hat. 
Für seine Strategie der Medien-Diskreditierung spielte es ihm in die Karten, dass zur Zeit des Wahlkampfs 2015 der Begriff »Fake News « breit diskutiert wurde. Insbesondere in der Online-Welt erschien eine Welle von Falschberichten und die Bezeichnung »Fake News« wurde als Genre für eine stark verzerrte oder völlig aus der Luft gegriffene Berichterstattung genutzt - ironischerweise auch aufgrund von bisweilen dubiosen Quellen, die Trumps Kandidatur unterstützen.

Mit dem Begriff »Fake News« generierte Trump eine große Aufmerksamkeit der Öffentlichkeit noch vor seiner Amtseinführung. Der $C N N$-Reporter Jim Acosta versuchte im Januar 2017 dem President Elect vehement eine Antwort abzuringen, indem er mit seiner Frage die Journalistin unterbrach, der Trump das Wort erteilt hatte. Trump wiederholte seine Ermahnung $»[\mathrm{~d}]$ on't be rude « mehrfach und schleuderte Acosta schließlich entgegen: »I am not gonna give you a question. You are Fake News « (Sutton, 2017). Mit dieser Anschuldigung löste Trump zwar nicht selbst einen Trend aus, aber verstärkte eine bestehende Tendenz vom Bully Pulpit ${ }^{8}$ aus massiv, wie eine Google-Trends-Analyse zeigt (Abbildung 1):

- fake news trump cnn fake news trump fake news awards

- is cnn fake news

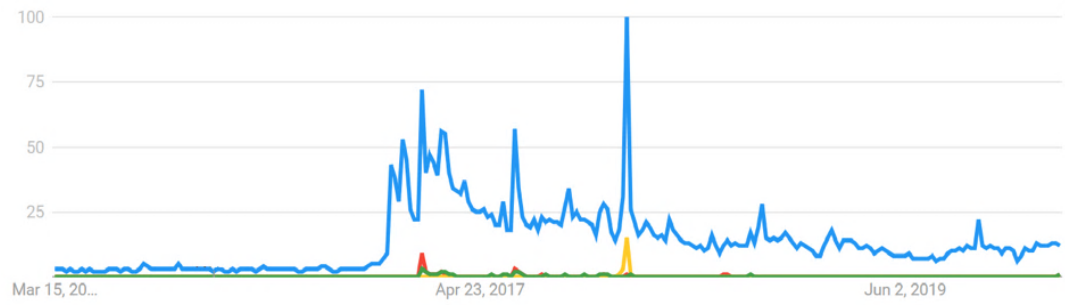

Abbildung 1: Google-Trends »Fake News«. Eigene Erhebung mittels Google Trends.

Die Zahlen der y-Achse stehen für das Suchinteresse relativ zum höchsten Punkt im Diagramm für die angegebene Zeit (x-Achse). Ein Wert von 100 ist die höchste Suchrate in Bezug auf einen Term oder Begriff. Eine Punktzahl von 0 bedeutet, dass für diesen Begriff nicht genügend Daten vorhanden waren. lichen Amtes oder seine Autorität, sich zu einem Thema öffentlichkeitswirksam zu äußern. 
Trump griff also lediglich einen bereits breit diskutierten Begriff auf und setzte ihn in einen neuen Kontext. Wenn er ihn jedoch in einem bestimmten Kontext nutzte, wurden wiederholt Höhepunkte bei den Suchanfragen erreicht, was auf eine große öffentliche Resonanz schließen lässt - und damit auch auf ein großes Potenzial für ein mögliches Reframing.

Ein Kommunikations-Frame beruht in der Regel auf einem Ausschnitt der Realität, es handelt sich dabei also um eine Form von erfahrbarer Wahrheit, die lediglich in eine spezifische, mitunter verzerrende Perspektive gesetzt wurde. Gewiss ist die große Mehrheit an Medienberichten keine »Fake News « und damit ist ein Framing mit jenem Begriff eigentlich schwierig. Wie zuvor erläutert, kamen jedoch Verzerrungen oder gar völlig aus der Luft gegriffene Berichte auch in etablierten Medien bereits vor. Trump nutzte diesen Ausschnitt der Realität und machte ihn zu einem neuen Hauptthema. In diesem Reframing verschob er mit seiner Zuschreibung über das »Fake News«-Label den Kontext von jenen Artikeln, die in alternativen Medien erschienen und richtete dieses Framing gegen jene, die das »Fake News«-Phänomen zunächst selbst breit diskutiert hatten: die etablierten Medien. Auf Basis des Misstrauens der ohnehin eher skeptischen konservativen Klientel konnte nun Trump nicht nur auf ein etwaiges Bias in der Berichterstattung verweisen, sondern grundsätzlich jedwede Nachricht, die ihm zuwider war, als falsch zurückweisen und sich selbst eine Opferrolle zuschreiben.

Die etablierten Medien hatten noch einen weiteren Anteil daran, dass sie Trump mit dem »Fake News «-Label derart kühn etikettieren konnte. Bereits in der heißen Phase des Wahlkampfes 2016 war die Seite klar, auf der sich die meisten großen Medienhäuser verorteten: 229 Tageszeitungen und 131 Wochenzeitschriften sprachen sich für Hillary Clinton aus. Lediglich neun Tageszeitungen und vier Wochenzeitschriften befürworteten Trump. Nicht nur zeigt dieses Verhältnis von 27:1, dass die Presse stark auf der demokratischen Seite steht, sie hatte sich auch von den Präferenzen eines großen Teils der Gesellschaft gelöst (Boczkowski, 2016). Es ist zwar nicht verwunderlich, dass sich viele Zeitungen gegen Trump aussprachen, schließlich verletzte er einige Normen der Politik und nicht wenige sahen in ihm eine Bedrohung für die Demokratie. Allerdings gab beispielsweise USA Today vorher noch nie eine Empfehlung für einen Präsidentschaftskandidaten ab - die Editoren sprachen sich diesmal jedoch gegen Trump aus (USA Today 2016). Zumindest die Maxime der politischen Unparteilichkeit des Mainstreams war spätestens damit überholt.

Noch eklatanter zeigt das Missverhältnis zwischen Trump und den Medien eine internationale Vergleichsstudie des Shorenstein Centers der 
Harvard Universität: Sender wie $C N N, N B C$ oder $C B S$ berichteten in über 90 Prozent der Berichterstattungen negativ über den Präsidenten. Das Feld führte hier zwar die deutsche $A R D$ mit 98 Prozent negativen Reportagen an, allerdings ließen wenige etablierte Medien in den USA ein gutes Haar an Trump (Patterson, 2017). In diesem Lichte ist es nicht verwunderlich, wenn Anhänger von Trump diesen Medien keinen Glauben mehr schenken wollen. Es ist schließlich vor allem Wut, welche die Akzeptanz von »Fake News« stark erhöht (Weeks, 2015). Entsprechend lügt Trump auch nicht unbedingt, wenn er mitunter Framings der Washington Post, von CNN oder der New York Times zurückweist, schließlich ist seine Perspektive stets auf einen anderen Fokus gerichtet - nämlich auf sein eigenes Framing. Dieses ist zwar oft auch verzerrt, nichtsdestoweniger steht Trumps Blickwinkel auf ein Thema so gut wie nie im Einklang mit dem Framing der MainstreamMedien.

In Bezug auf den Begriff der »Fake News « ist das Framing eines Sachverhalts tatsächlich die Krux. Die Überprüfung der Fakten ist zwar relativ einfach, die Frage nach deren Bedeutung, politischen Implikationen und Verantwortungen kann jedoch nicht auf Mechanismen der automatischen Bestätigung reduziert werden (Coleman, 2018). Hier ist es nach wie vor das Medien-Framing, das Probleme entsprechend definiert und in Kontexte setzt, das Verantwortungen zuweist und moralische Urteile fällt. Auch jene Setzungen können als falsch eingestuft werden, wenn sie nicht mit dem eigenen Verständnis korrespondieren.

Weiterhin gab es tatsächlich einzelne Vorkommnisse, bei welchen die Mainstream-Medien Falschinformation über Trump verbreiteten. So berichtete Zeke Miller, ein Reporter des Time Magazines, beispielsweise, dass Trump eine Büste von Martin Luther King Jr. aus dem Oval Office entfernen hat lassen (Gibbs, 2017). Dies war eine Falschmeldung, die zeitnah korrigiert wurde, Trump stufte sie jedoch als vorsätzlich falsche Berichterstattung ein. Selbst auf Politifact wurden bereits Vorwürfe Trumps gegenüber der Presse bestätigt, so stufte das Fact-Checking-Portal beispielsweise jene Berichte, dass Trump im Jahr 2016 eine Mutter mit einem (schreienden) Kind aus einer Veranstaltung verwies, als falsch ein (Politifact, 2016). Diese vereinzelten Fehler oder gar vorsätzliche Desinformationen bestätigen nicht nur Trumps »Fake News«-Vorwürfe; es zeigte sich auch, dass 46 Prozent der Amerikaner davon überzeugt sind, dass die Presse Berichte über Trump erfindet (Politico, 2017).

Die Feindschaft zwischen der Presse und der Trump-Regierung dokumentiert auch ein Disput um einen Werbespot: Eine Interessengruppe wollte mit diesem Werbefilm Trumps Errungenschaften der ersten 100 
Tage Amtszeit anpreisen. Große Medienorganisationen, darunter $C N N$, $N B C, A B C$ und $C B S$, lehnten die Ausstrahlung des bezahlten Werbespots jedoch ab. Die Kampagnensprecher beklagten einen »chilling precedent against free speech rights « (Donaldjtrump.com, 2017). Hannity kommentierte auf Fox News:

It's a great ad and it highlights all the wonderful things that have happened...It's really disappointing...this is supposed to be a free society. We have freedom of speech. The fact that this ad is not being shown on $C N N$, on $N B C$, on $C B S$, on news networks who have a duty to report to the public the facts...is really, really ridiculous to me. It's really sad. (Bedard, 2017)

Ein großes öffentliches Interesse weckte auch Trumps Lancierung der Fake News Awards im Jahr 2017 (Google Trends, 2019). Der Präsident verlieh den Preis zumeist an CNN und die New York Times, aber auch die Washington Post und Newsweek landeten unter den Top 10. Den Höhepunkt der Auseinandersetzung zwischen Trump und den etablierten Medien bildet jedoch womöglich sogar sein Retweet eines Videos im Juli 2017. In diesem schlägt Trump in einer Wrestling-Show einen Kontrahenten nieder, am Kopf des Gegners wurde im Video jedoch ein $C N N$-Logo eingefügt. Trump erntete harsche Kritik für den Re-Post sowie den Vorwurf, Gewalt zu billigen; schließlich brachte nur etwas über einen Monat zuvor Greg Gianforte, ein republikanischer Kandidat für das Abgeordnetenhaus, bei einer Begrüßungsveranstaltung in seinem Büro einen Journalisten des Guardian zu Boden und zerbrach dabei seine Brille (Keneally, 2017). Trump lobte indirekt sein Verhalten mit den Worten »any guy who can do a body slam, he is my type! « (Keneally, 2017). Auch die Klientel von Gianforte scheint zumindest durch seine Attacke nicht abgeschreckt worden zu sein: Er wurde kurz darauf im Amt bestätigt.

Auch mit CNNs Acosta kam es im weiteren Verlauf von Trumps Präsidentschaft zu wiederholten Disputen. Im Februar 2019 entgegnete Trump dem $C N N$-Reporter hinsichtlich einer kritischen Frage zu seiner Migrationspolitik: "You have an agenda, you are CNN, you are Fake News « (Fox News, 2019). Die Verwendung dieses Framings schafft eine Normalisierung im öffentlichen Diskurs und diese drei kurzen Sätze verweisen $C N N$, faktisch aber eine ganze Sparte, in die politisch liberale Ecke, womit sie für viele Beobachter diskreditiert sind. Dies hat bei Trump mittlerweile System, denn auch in seinem Wahlkampf für die Kandidatur des Jahres 2020 schießt sich Trump auf die etablierten Medien ein:

Michael,

The FAKE-NEWS media has spent the last two years LYING to the American people and trying to take me down. 
They are so blinded by their anti-Trump agenda that they have decided to completely disregard FACTS and instead choose to continue peddling their lies and conspiracy theories. They are truly a threat to our Democracy.

Outlets like CNN can't accept the fact that the WITCH HUNT they pushed for two years turned out to be a total farce. No wonder their ratings are so terrible! [...]

We need to let the mainstream media know that we are FINISHED putting up with their constant lies and attacks.

The duty of the media is to accurately inform the public, and our current national media has COMPLETELY FAILED in that regard.

(Donald J. Trump, persönliche Kommunikation 05.07.2019, 18:05 MEZ)

Diese Medien-Strategie scheint Erfolg zu haben. Mittlerweile schätzen Amerikaner, dass gar 44 Prozent der vorhandenen Nachrichten »Fake News« seien (Jones, 2018). In der Forschung zeigte sich darüber hinaus, dass für einige als »Fake News« auch einfach jene Nachrichten gelten, deren Inhalt sie keinen Glauben schenken mögen. Die ideologische Voreingenommenheit ist also bereits so weit fortgeschritten, dass sie selbst die Abgrenzung von Fakten und Überzeugungen verwischt (Nielsen \& Graves, 2017). Mit dieser Heuristik schenken Rezipienten einer gefühlten Wahrheit mehr Glauben als über etablierte Medien berichtete Fakten. Bis vor zehn Jahren war dies noch ein Fall für die politische Satire. Stephen Colbert führte in der Pilotsendung seiner Satireshow über Konservative und Republikaner, der Colbert Report, den Begriff Truthiness ein. Merriam-Webster (2019) definiert den Begriff folgendermaßen: »Truthiness refers to the quality of seeming to be true but not necessarily or actually true according to known facts. "Colbert führt in einem Interview weiter aus: »Truthiness is 'What I say is right, and [nothing] anyone else says could possibly be true.' It's not only that I feel it to be true, but that $I$ feel it to be true. There's not only an emotional quality, but there's a selfish quality« (Rabin, 2006). Auch dies ist Teil der neuen Heuristik vieler Menschen aus dem konservativen Spektrum, die vor allem Trumps Rhetorik folgen.

\section{Fazit}

Trump ist es gelungen, die Glaubwürdigkeit der etablierten amerikanischen Medien unter seinen Anhängern relativ weitreichend zu eliminieren. Er hatte dafür den Begriff der »Fake News«, das Misstrauen gegenüber dem Mainstream und die Ablehnung seiner Person in vielen dieser Publikationen fusioniert - das Ergebnis konnte er geballt gegen die Medien einsetzen. Durch dieses Strategem erlangte er einen großen Anteil der Deutungshoheit 
über die Realität, schließlich ist der Term »Fake News« auch ein Abgrenzungsbegriff, der die Existenz einer Wahrheit impliziert. Diese sei jedoch nie in den Mainstream-Medien zu finden. Deren Framing ermöglichte das Framing mit dem »Fake News«-Begriff, da Interpretationen mit einem eher progressiven Bias dort eher vorkommen als ein konservativerer Standpunkt. Auch wenn die Mainstream-Medien in der Regel keine Fantasiegeschichten publizieren, ist ihnen aus Sicht der Konservativen zumindest ein gefühltes progressives Bias inhärent.

Da ein Teil der amerikanischen Gesellschaft bereits über längere Zeit hinweg eine hohe Skepsis gegen die etablierte Presse hegte, konnte Trump den verbliebenen Teil ihrer Integrität angreifen. Infolgedessen brach das Vertrauen der Skeptiker in die Glaubwürdigkeit der etablierten Nachrichtenmedien komplett ein. Ohne jene Skepsis und eine Tendenz zur Progressivität wäre der »Fake News«-Vorwurf niemals so erfolgreich geworden. Ein Teil der amerikanischen Bevölkerung lehnt den progressiven Pfad, den die USA in den letzten Jahrzehnten beschritten hat, so entschieden ab, dass er alles ihm - auch nur gefühlt - zugehörige ausblendet und als grundfalsch zurückweist. Aufgrund dieser Haltung ist zu urteilen, dass selbst wenn sich Journalisten um bestmögliche Neutralität bemühen, die Einschätzung über die skeptische Heuristik ein Bias vermuten würde. Nicht zuletzt hatte die überdurchschnittlich negative Berichterstattung über Präsident Trump einen Anteil daran, gerade weil Trump eine extrem hohe Beliebtheit unter seinen Anhängern erfährt. Da sie dem auch keinen Glauben schenken wollen, verwischt die Grenze zwischen Fakt und Überzeugung. Damit ist das Framing mit dem »Fake News«-Begriff als Label zwar auch ein Symptom einer größeren Krise, die von Misstrauen gegenüber verschiedenen Institutionen geprägt ist; spezifisch für die USA ist sie jedoch Teil einer ideologischen Verhärtung, die auf beiden Seiten weniger Kompromiss zulässt und vor allem den Blick auf die jeweils andere Seite stark einschränkt.

Neben Donald Trump existieren freilich auch andere Politiker, die sich dem »Fake News«-Label zahlreich bedienen. Beispielsweise hatte der ecuadorianische Präsident Rafael Correa die Medien als seinen größten Feind bezeichnet und auch im Umfeld der PEGIDA-Bewegung (Patriotische Europäer gegen die Islamisierung des Abendlandes) wurde der Vorwurf der Lügenpresse häufig genutzt. Bislang wurde dies in Deutschland allerdings noch zu keinem systemischen Phänomen. Dennoch ist die derzeitige Entwicklung derer in den USA nicht unähnlich. Auch in Deutschland kehren Menschen vermehrt den etablierten Medien den Rücken zu. Auch hier sind bei einigen Medienhäusern sozialliberale Tendenzen zu erkennen und der Vorwurf, dass die Öffentlich-Rechtlichen voreingenommen berichten, hält 
sich hartnäckig, obgleich dies nicht durchgängig der Fall ist. Nichtsdestoweniger tragen sowohl die starke Ablehnung Trumps bei Sendern wie der $A R D$ als auch generell Berichterstattungen, die eine politische Agenda vermuten lassen, zur Polarisierung einer Gesellschaft bei. Aus dieser Reaktion heraus ist abzulesen, dass retrospektiv die Maxime der alten journalistischen Garde vielleicht gar nicht so verkehrt war: Walter Cronkite verknüpfte das Vertrauen der Menschen in die Medien mit einer einfachen Logik: »In seeking truth, you have to get both sides of a story« (Felling, 2007). Ein noch so geringes Bias kann es möglich machen, dass eine Seite keine Wahrheit in einer Darstellung mehr erkennt. So kann das Unterlassen von Hanns Joachim Friedrichs journalistischem Credo »Mache dich mit keiner Sache gemein« einen Teil der Gesellschaft von Medien mit eigentlich zentristisch-ausgewogener Berichterstattung vergraulen und einem verzerrten Mediensektor zuspielen. Das hat Effekte bis hin zu einer Fragmentierung der Öffentlichkeit sowie Absolutierung von Meinungen hin zu Wahrheiten. Für jenen Teil, der sich von den Mainstream-Medien losgelöst hat, wird jedoch jeder Versuch von mehr Ausgeglichenheit zu spät kommen. Deren Heuristik ist umgeschrieben.

\section{Literatur}

Allcott, Hunt \& Gentzkow, Matthew (2017). Social Media and Fake-News in the 2016 election. Journal of Economic Perspectives, 31(2), 211-235.

Aufderheide, Patricia (1990). After the Fairness Doctrine. Controversial Broadcast Programming and the Public Interest. Journal of Communication, 40 (3), 47-72.

Bateson, Gregory (1972). A Theory of Play and Fantasy. In: Bateson, G. (Hrsg.), Steps to an Ecology of Mind (177-193). New York: Ballantine Books.

Bedard, Paul (2017). CNN, ABC, CBS and NBC ban Trump 100 Days ad. Washington Examiner, 05. Mai 2017. Abgerufen von https://www.washingtonexaminer.com/cnnabc-cbs-and-nbc-ban-trump-100-days-ad.

Bennett, Lance \& Livingston, Steven (2018). The Disinformation Order. Disruptive Communication and the Decline of Democratic Institutions. European Journal of Communication, 33(2), 122-139. DOI: 10.1177/0267323118760317.

Boczkowski, Pablo J. (2016). Has Election 2016 Been a Turning Point for the Influence of the News Media? Nieman Lab, 8. November 2016. Abgerufen von http://www.niemanlab.org/2016/11/has-election-2016-been-a-turning-point-for-theinfluence-of-the-news-media/.

Buckley, William (1955). Mission Statement. National Review, 1(1).

Chen, Serena \& Chaiken, Shelly (1999). The Heuristic-Systematic Model in its Broader Context. In: Chaiken, S. \& Y. Trope (Hrsg.), Dual-Process Theories in Social Psychology (73-96). New York: Guilford Press. 
Coleman, Steven (2018). The Elusiveness of Political Truth. From the Conceit of Objectivity to Intersubjective Judgement. European Journal of Communication, 33(2), 157-171. DOI: 10.1177/0267323118760319.

Donaldjtrump.com (2017). All Mainstream TV Networks Block Paid Campagn Ad Setting a Chilling Precedent Against Free Speech. Abgerufen von https://www. donaldjtrump.com/media/all-mainstream-tv-networks-block-paid-campaign-ad-setting-a-chilling-preced.

Egelhofer, Jana L. \& Lecheler, Sophie (2019). Fake News as a Two-Dimensional Phenomenon. A Framework and Research Agenda. Annals of the International Communication Association, 43(2), 97-116. DOI: 10.1080/23808985.2019.1602782.

Entman, Robert M. (1993). Framing. Toward Clarification of a Fractured Paradigm. Journal of Communication, 43(4), 51-58. DOI: 10.1111/j.14602466.1993.tb01304.x.

Entman, Robert M. \& Usher, Nikki (2018). Framing in a Fractured Democracy. Impacts of Digital Technology on Ideology, Power and Cascading Network Activation. Journal of Communication, 68 (2), 298-308. DOI: 10.1093/joc/jqx019.

Feldman, Lauren (2011). Partisan Differences in Opinionated News Perceptions. A Test of the Hostile Media Effect. Political Behavior, 33(3), 407-432. DOI: 10.1007/s11109-010-9139-4.

Felling, Matthew (2007). From The Vault. Cronkite \& Hoffa. CBS News, 14. September 2007. Abgerufen von https://www.cbsnews.com/news/from-the-vault-cronkitehoffa/.

Fox News (2019). Trump spars with CNN's Acosta. You have an agenda, you're fake news. Abgerufen von https://www.youtube.com/watch?v=LxESGtZwUi0.

Gibbs, Nancy (2017). A Note to Our Readers. Time, 24. Januar 2017. Abgerufen von https://time.com/4645541/donald-trump-white-house-oval-office/.

Goffman, Erving (1974). Frame analysis. Boston: Northeastern University Press.

Google Trend (2019). Abgerufen von https://www.google.com/trends/explore?q= fake $\% 20$ news.

Guardino, Matt \& Snyder, Dean (2012). The Tea Party and the Crisis of Neoliberalism. Mainstreaming New Right Populism in the Corporate News Media. New Political Science, 34(4), 527-548. DOI: 10.1080/07393148.2012.729741.

Hahn, Kyu S. \& Iyengar, Shanto (2009). Red Media, Blue Media. Evidence of Ideological Selectivity in Media Use. Journal of Communication, 59(1), 19-39. DOI: 10.1111/j.1460-2466.2008.01402.x.

Harvard CAPS \& Harris Poll (2017). Harvard's Center for American Political Studies (CAPS) and Harris Insights and Analytics. Abgerufen von https://harvard harrispoll.com/wp-content/uploads/2017/05/Harvard-Harris-Poll_May-Wave_ 05.26.2017_TECH.pptx.

Hermida, Alfred (2011). Fluid Spaces, Fluid Journalism. The Role of the »Active Recipient« in Participatory Journalism. In: Singer, J. B.; A. Hermida; D. Domingo et al. (Hrsg.), Participatory Journalism. Guarding Open Gates at Online Newspapers (177-191). Malden, MA: John Wiley \& Sons. 
James, William (1998 (1890)). The principles of psychology. New York, NY: Henry Holt and Co.

Jones, Jeffrey M. (2018). Americans. Much Misinformation, Bias, Inaccuracy in News. Abgerufen von https://news.gallup.com/opinion/gallup/235796/americans-misinfor mation-bias-inaccuracy-news.aspx.

Keneally, Meghan (2017). What happened when Republican Greg Gianforte bodyslammed a reporter. $A B C$ News, 19. Oktober 2018. Abgerufen von: https://abcnews.go.com/Politics/happened-republican-greg-gianforte-bodyslammed-reporter/story?id=58610691.

Korten, David C. (2001). When Corporations Rule the World. West Hartford: Kumarian Press.

Landau, Mark J.; Keefer, Lucas A. \& Rothschild, Zachary K. (2014). Epistemic motives moderate the effect of metaphoric framing on attitudes. Journal of Experimental Social Psychology, 53, 125-138. DOI: 10.1016/j.jesp.2014.03.009.

Limbaugh, Rush (2009). Climate Gate Hoax. The Universe of Lies Versus the Universe of Reality. Abgerufen von http://www.rushlimbaugh.com/daily/2009/11/24/climate gate_hoax_the_universe_of_lies_versus_the_universe_of_reality.

Link, William A. (2013). Time is an Illusive Companion. In: Shermer, T. (Hrsg.), Barry Goldwater and the Remaking of the American Political Landscape (238-258). University of Arizona Press.

Lischka, Juliane A. (2019). A Badge of Honor? Journalism Studies, 20(2), 287-304. DOI: $10.5167 /$ uzh-142117.

Major, Mark (2012). Objective but Not Impartial. Human Events, Barry Goldwater, and the Development of the »Liberal Media« in the Conservative Counter-Sphere. New Political Science, 34(4), 455-468. DOI: 10.1080/07393148.2012.729737.

Marchi, Regina (2012). With Facebook, Blogs, and Fake News, Teens Reject Journalistic »Objectivity«. Journal of Communication Inquiry, 36(3), 246-262. DOI: $10.1177 / 0196859912458700$.

Meraz, Sharon (2011) Using Time Series Analysis to Measure Intermedia Agenda-Setting Influence in Traditional Media and Political Blog Networks. Journalism \& Mass Communication Quarterly, 88(1), 176-194. DOI: 10.1177/107769901108800110.

Merriam-Webster (2019). 'Truthiness'. Can Something »Seem« Without Being, True? When you just know. Abgerufen von https://www.merriam-webster.com/words-atplay/truthiness-meaning-word-origin.

Metzger, Miriam J.; Flanagin, Andrew J. \& Medders, Ryan B. (2010). Social and Heuristic Approaches to Credibility Evaluation Online. Journal of Communication, 60 (3), 413-439. DOI: 10.1111/j.1460-2466.2010.01488.x.

Nash, George H. (2006). The conservative intellectual movement in America since 1945. Wilmington: ISI Books.

Nielsen, Rasmus K. \& Graves, Lucas (2017) »News you don't believe«. Audience perspectives on fake news. Abgerufen von https://reutersinstitute.politics.ox.ac.uk/ sites/default/files/2017-10/Nielsen\&Graves_factsheet_1710v3_FINAL_download.pdf. 
Nielsen Media Research (2013). Evening Network News Share over Time. Abgerufen von http://www.journalism.org/media-indicators/evening-net-work-news-shareover-time.

Oswald, Michael (2018). Die Tea Party als Obamas Widersacher und Trumps Wegbereiter. Strategischer Wandel im Amerikanischen Konservatismus. Wiesbaden: Springer VS.

Patterson, Thomas E. (2017). News Coverage of Donald Trump's First 100 Days. HKS Working Paper No. RWP17-040. DOI: 10.2139/ssrn.3040911

Peck, Reece (2014). »You Say Rich, I Say Job Creator«. How Fox News Framed the Great Recession Through the Moral Discourse of Producerism. Media, Culture \& Society, 36 (4), 526-535. DOI: 10.1177/0163443714527565.

Politico (2017). Poll. 46 percent think media make up stories about Trump. Abgerufen von https://www.politico.com/story/2017/10/18/trump-media-Fake-News-poll243884.

Politifact (2016). Donald Trump accurately says media wrong that he kicked baby out of rally. Abgerufen von https://www.politifact.com/truth-o-meter/state ments/2016/aug/08/donald-trump/donald-trump-accurately-says-media-wrong-hekicked/.

Prior, Markus (2013). Media and Political Polarization. Annual Review of Political Science, 16, 101-127. DOI: 10.1146/annurev-polisci-100711-135242.

Rabin, Nathan (2006). Interview. Steven Colbert. The A.V. Club, 25. Januar 2006. Abgerufen von https://tv.avclub.com/stephen-colbert-1798208958.

Richardson, Nick (2017). Fake News and Journalism Education. Asia Pacific Media Educator, 27(1), 1-9. DOI: 10.1177/1326365X17702268.

Sutton, Kelsey (2017). Trump Calls CNN 'Fake-News,' as Channel Defends its Reporting on Intelligence Briefing. Politico, 11. Januar 2017. Abgerufen von https://www.politico.com/blogs/on-media/2017/01/trump-refusing-to-answer-ques tion-from-cnn-reporter-you-are-Fake-News-233485.

Tandoc, Edson C. \& Vos, Tim P. (2016). The Journalist is Marketing the News. Social Media in the Gatekeeping Process. Journalism Practice, 10(8), 950-966. DOI: 10.1080/17512786.2015.1087811.

Tandoc, Edson C.; Lim, Zheng Wei \& Ling, Richard (2018). Defining »Fake News«. Digital Journalism, 6(2), 137-153. DOI: 10.1080/21670811.2017.1360143.

Tandoc, Edson C.; Ling, Richard; Westlund, Oscar; Duffy, Andrew; Goh, Debbie \& Zheng Wei, Lim (2017). Audiences' acts of authentication in the age of fake news. A conceptual framework. New Media \& Society, 20(8), 2745-2763. DOI: $10.1177 / 1461444817731756$.

Thomsen, Jacqueline (2017). Trump: I 'really started this whole Fake-News thing'. The Hill, 25. Oktober 2017. Abgerufen von https://thehill.com/homenews/administra tion/357225-trump-i-really-started-this-whole-Fake-News-thing.

USA Today (2016). USA Today's Editorial Board: Trump is 'Unfit for the Presidency'. Abgerufen von https://eu.usatoday.com/story/opinion/2016/09/29/dont-vote-fordonald-trump-editorial-board-editorials-debates/91295020/. 
Vargo, Chris \& Guo, Lei (2017). Networks, big data, and intermedia agenda-setting. an analysis of traditional, partisan, and emerging online U.S. news. Journalism \& Mass Communication Quarterly. Online Vorveröffentlichung. DOI: 10.1177/ 1077699016679976.

Weeks, Brian E. (2015). Emotions, Partisanship, and Misperceptions. How Anger and Anxiety Moderate the Effect of Partisan Bias on Susceptibility to Political Misinformation. Journal of Communication, 65(4), 699-719. DOI: 10.1111/jcom.12164.

Williams, Rhys H. (2004). The Cultural Contexts of Collective Action. Constraints, Opportunities, and the Symbolic Life of Social Movements. In: Snow, D. A.; S. A. Soule \& H. Kriesi (Hrsg.), The Blackwell Companion to Social Movements (91-115). Oxford: Blackwell Publishing Ltd. 
